\title{
A microcomputer-based tracking task for examination of the spatial and temporal organization of perceptual motor skill
}

\author{
G. GARDINER and I. M. FRANKS \\ The University of British Columbia, Vancouver, British Columbia, Canada \\ and \\ DAVID GOODMAN \\ Simon Fraser University, Burnaby, British Columbia, Canada
}

\begin{abstract}
A microcomputer-based tracking task is described in detail. The program that controls this task was written in Pascal and 6502 assembler language and was designed to run on an Apple IIe computer. The program was designed to allow the experimenter to vary such parameters as the velocity of the stimulus signal. In addition, several indicants of performance are recorded simultaneously and are averaged over selected time intervals during each trial. The paper also illustrates the applications of this particular computer program in the study of motor control and learning.
\end{abstract}

To facilitate the study of perceptual motor skill learning, we developed a generalized microcomputer-based program that controls a tracking task. This task was adapted in part from Pew's (1966) compensatory tracking task, in which he investigated the hierarchical control of action systems. The generalized nature of the task allows for variations in the stimulus signal and the selection of derived error scores. In addition to determining the control system variables, the program can also determine the number of trials, the tracking time per trial, and the time course over which the derived error scores can be taken.

The program, written in Apple PASCAL and 6502 assembler language, was designed to run on the Apple IIe with a Grappler + printer interface card. For greater efficiency, the buffered version of the Grappler card should be used. In all versions, the objective of the task is to control the movements of a target cursor (presented on the computer video terminal) using two response keys from the keyboard. Depression of these two keys by the right and left index fingers results in movement of the target cursor.

\section{DESCRIPTION OF THE TRACKING PROGRAM FOR THE USER}

To start the program, the user places the diskette into drive 1 and switches the computer on. The program is then automatically loaded into memory, and execution begins. Several title pages are displayed, and the user enters administrative details (e.g., the subject's name and the

The computer program was written with the aid of NSERC grant A0434. Please address correspondence to I. M. Franks, 6081 University Blvd., University of British Columbia, Vancouver, British Columbia V6T IW5, Canada. date of the trial). The user is informed that keys $J$ and $\mathrm{F}$ are used to control the target cursor's movement.

\section{Stimulus Signal}

The program queries the user as to the characteristics of the stimulus signal. Several lines are displayed on the screen to indicate the region in which the target cursor must be kept. The larger the region, the easier the task. Selection of "zero" region produces a true compensatory tracking task that requires the subject to maintain the target cursor position over a single center line. Another option is that the experimenter may want the subject to use the boundary lines as positions of reversal. That is, the subject's task is to reverse direction of the cursor (by depression of either the $\mathrm{J}$ or the $\mathrm{F}$ key) as close to these boundary lines as possible. (Error scores are possible for this condition.) Thus, the subject produces a cyclical response function using a rhythmical tapping pattern.

If selection of a region greater than zero is made, another option is available. An alternating line can be placed anywhere within the boundaries of the bandwidth. When the alternating line appears, the subject's task is to reverse direction of the target cursor at the position of the alternating line. This feature has the effect of varying the complexity of the tapping task and, hence, the produced response function. If no alternating line is desired, then a "zero" option is entered into the computer.

\section{Response Control}

The speed of the cursor movement varies by a factor of $11 \mathrm{~mm} / \mathrm{sec}$, and is displayed on a standard computer video monitor. The program displays a series of lines that indicate how far the cursor will travel in a period of $1 \mathrm{sec}$ at a constant velocity. 
At the commencement of each trial, the target cursor moves at constant velocity to the left or right and simulates an unbalanced system. Depression of either the $J$ or the F key immediately reverses the direction of the cursor.

\section{Procedure}

A warning tone occurs $5 \mathrm{sec}$ prior to each trial, and the target cursor flashes during this foreperiod. The trial begins and continues for a preselected time period, and performance measures are computed and averaged over various time intervals. For example, if a trial length is $30 \mathrm{sec}$, it is possible to average error scores over time periods within that 30 -sec period (i.e., every $5 \mathrm{sec}$, $10 \mathrm{sec}$, etc.). After each trial, it is possible to provide results, or to control the intertrial interval and send the results to the screen, printer, or diskette at the end of the experimental session.

\section{Selection of Derived Error Scores}

Either or all of three possible performance displays can be sent to the screen or the printer or can be saved on diskette. The scatter plot places a dot at the exact location of the cursor when a response key is pressed during the trial (see Figure 1). This plot collapses all responses and does not take into accuont the time of response. A second plot includes time and uses the printer or screen as a strip chart recorder. The response function that has been produced is graphed over time (see Figure 2). Numerical error analyses give the variable, absolute, and constant error about the selected boundary lines, in addition to averaging these values for an overall integrated error score. All of these measures are partitioned into preselected time intervals within the trial and are shown in Table 1. A buffer of $10 \mathrm{sec}$ should be added to each trial in order to allow accurate and reliable measures when the time partition is used. The ESC key cancels the performance measure display in progress and returns control to the experimenter to start the trial again.

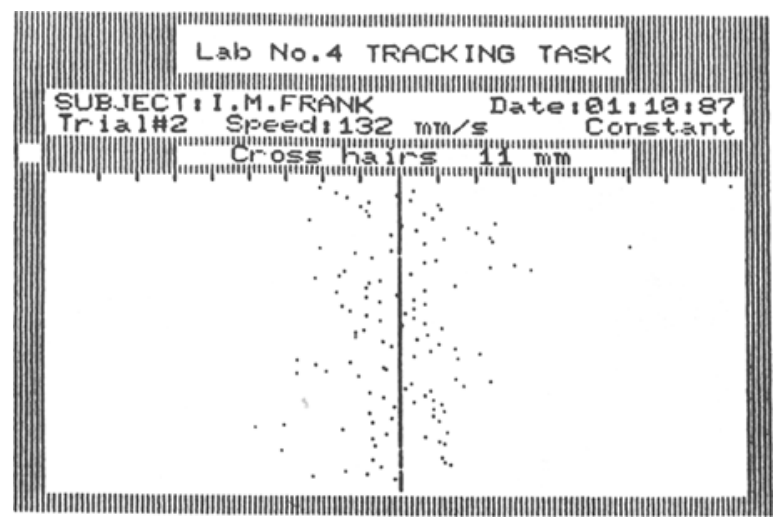

Figure 1. An example of a scatter plot in which the results are collapsed over time. Each point represents a depression of either a $J$ or an $F$ response key.

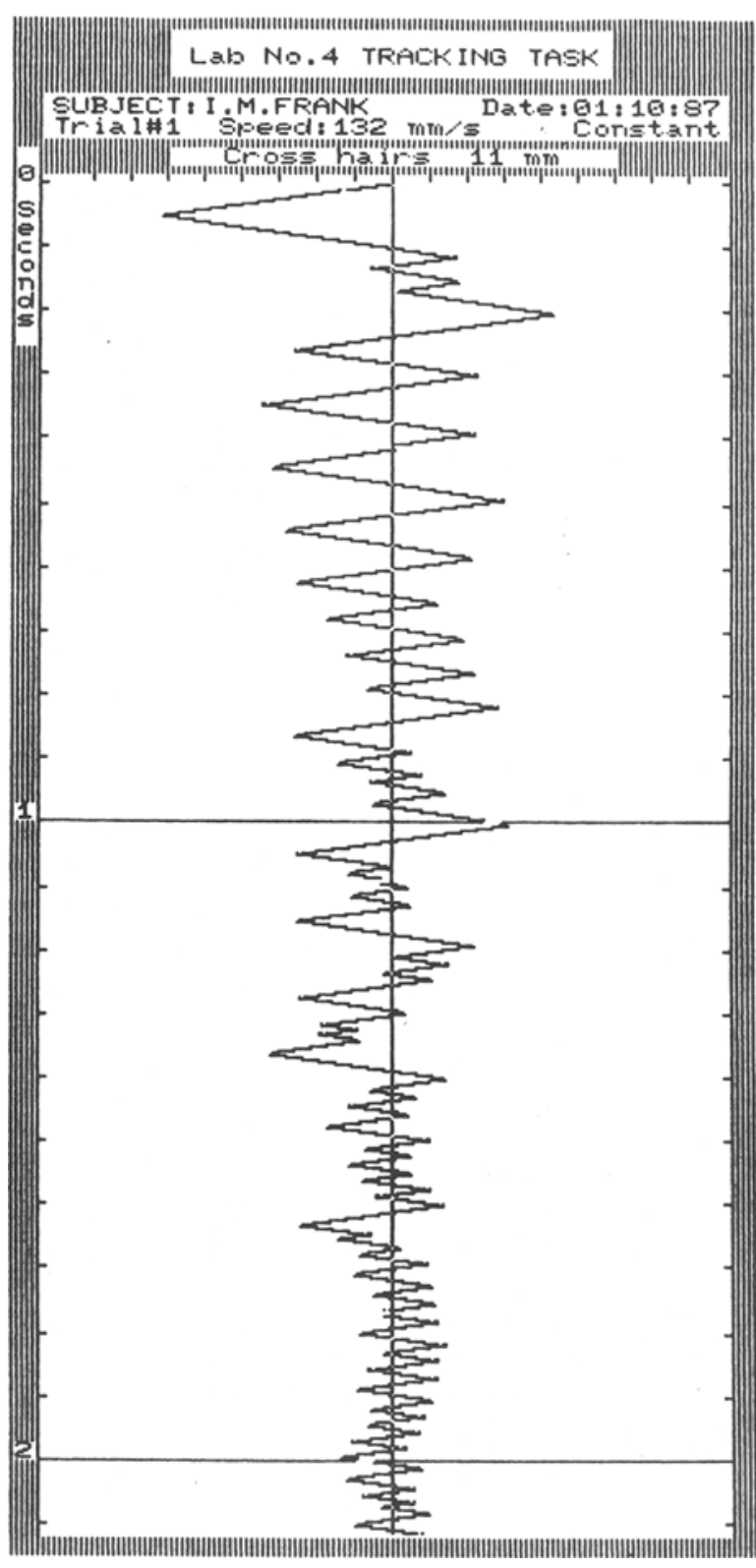

Figure 2. An example of a response function that was produced during a true compensatory tracking task.

\section{THE COMPUTER PROGRAM}

The control program is a combination of Apple ASCAL 1.2 interfaced with 6502 assembler language. The assembly routines are used for low-level control where speed and timing are essential. The main program also uses three units in the PASCAL SYSTEM LIBRARY; therefore, that file must also remain on the boot disk. Figure 3 illustrates the flow of control between modules.

The tasks of the secondary modules are as follows: 
Table 1

Example of the Display of Error Scores that Can be Printed to Screen or Printer After Each Trial

\begin{tabular}{ccc}
\hline & $\begin{array}{c}\text { Summary Scores for I. M. Franks: } \\
\text { (Trial } \# 2 \text { Speed }=132 \mathrm{~mm} / \mathrm{sec} \text { ) }\end{array}$ \\
Time & Error & Score \\
\hline 0 & Absolute & 10.3 \\
& Constant* & -2.4 \\
9 & Variable & 20.8 \\
10 & Absolute & 7.5 \\
19 & Constant & 5.2 \\
Total & Variable & 10.1 \\
& Absolute & 17.8 \\
& Constant & 2.8 \\
& Variable & 30.9 \\
\hline
\end{tabular}

Note-The 20-sec trial was partitioned into 10-sec intervals. All values are calculated in millimeters. ${ }^{*} A$ negative value refers to bias to the left of the center criterion line.

File 1: MAINREACT.TEXT (PASCAL)

\subsection{BORDER}

-Draws the border around the video screen and labels the display.

-Reads in the subject's name and date of trial.

\subsection{SETPARMS}

-Initializes: (a) the width of the target lines:

(b) the position of the alternate line:

(c) the speed of the cursor:

File 2: ASSEM.TEXT (PASCAL)

\subsection{REACT}

-Controls the speed and direction of the target cursor.

-Records the cursor position when a response key is pressed.

-Causes the alternate line to blink.

File 3: GRAPHICS.TEXT (6502)

\subsection{GRAPH}

-Graphs all the data on the screen.

-Calculates the error score.

-Sends data to the printer.

File 4: UP.TEXT (6502)

\subsection{SCROLL}

-Scrolls the screen to simulate a chart recorder.

\section{Timing Calculations}

All time-dependent control is performed by the subroutine REACT. This module is written in 6502 assembler so that the exact execution time of the object code can be determined in clock cycles. The most critical timedependent function is the control of the cursor speed across the video screen. The velocity is calculated using high-resolution graphic dots/second, where 14 dots represent $1 \mathrm{~mm}$. Since one pass through the main control loop of REACT advances the cursor by one dot position, velocity control can be achieved by adjusting a time delay at the end of this main program loop. To protect timing integrity, all conditional branches within the assembler code result in the same number of clock cycles, regardless of the outcome at the branch.

To adjust the time delay, the speed (v) parameter (set by the user in the PASCAL section) is used. This speed
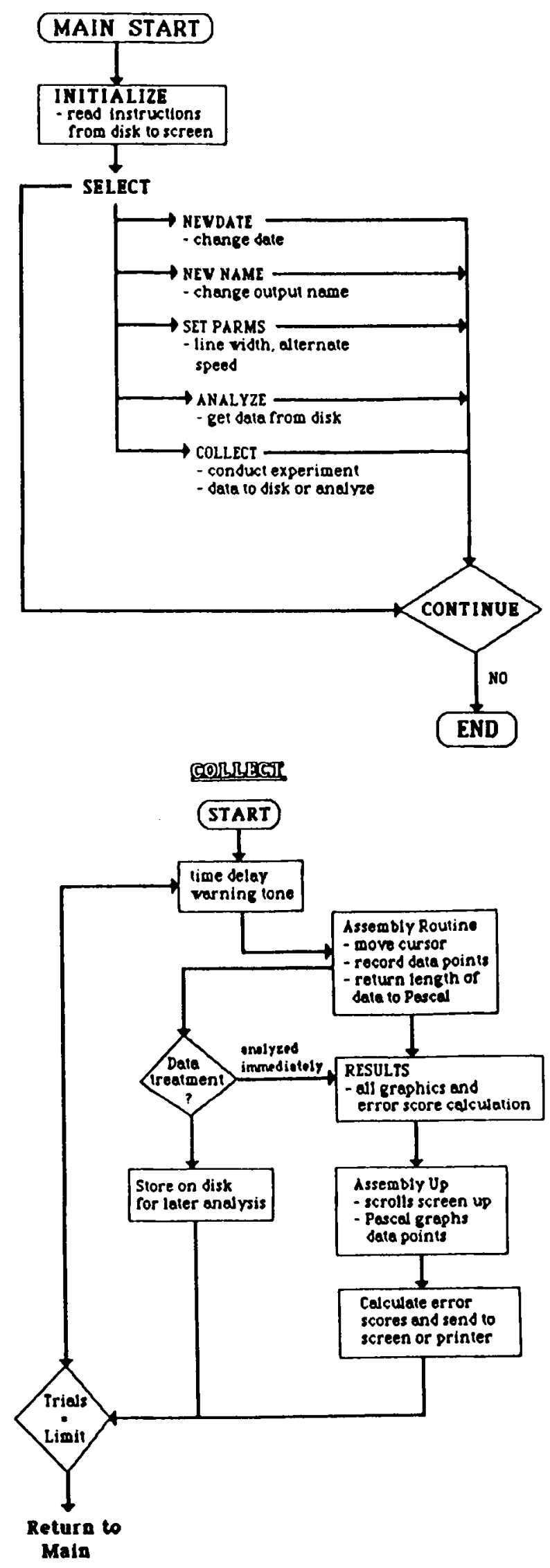

Figure 3. The flow of control between modules in the tracking program. The bottom flow chart details the "collect" subprogram. 
parameter is converted to dots/second before being passed to REACT. In the delay code, a constant $(K)$, which relates to the 1.023-MHz Apple clock rate, is successively decremented by the value of the speed parameter until zero is reached, at which time the main loop repeats and the cursor is written to the next dot position. Larger values of the speed parameter result in a smaller delay since zero is reached much faster.

The constant is derived from the following:

$$
\begin{aligned}
K & =[1.023 \mathrm{MHz}-(v \text { dots } / \mathrm{sec} \times 659 \mathrm{cycles} / \mathrm{dot}) / 33] \\
& : K=31000-20 v
\end{aligned}
$$

where 33 is the number of clock cycles to execute the delay code once, and 659 is the number of clock cycles to execute the main loop excluding delay.

A simplified version of the algorithm is as follows:

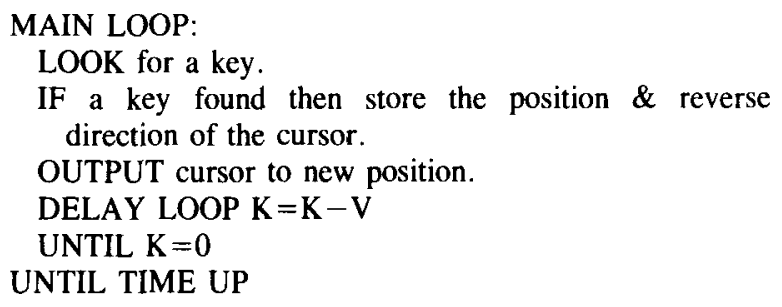

Examination of this simplified algorithm presents several constraints: (1) There is a small hardware time delay between the striking of a key and placing the key in the keyboard buffer. This time delay is dependent on the scan rate and debounce time (see Apple II Reference Manual for the IIe, p. 164). (2) The video refresh rate is set at $60 \mathrm{~Hz}$. Consequently, the cursor image, written directly to the display memory, may take up to $17 \mathrm{msec}$ before it appears on the video display screen and, on average, is delayed $8 \mathrm{msec}$. (3) The change in cursor direction is an instantaneous change in the opposite direction with a constant velocity. (4) The majority of time is spent in the DELAY LOOP, which controls the cursor speed. During this time the keyboard has no effect.

The following formula is used to derive the time between response keystrokes. This time variable is used by the graphics routine:

$$
\text { Time }=\mathrm{HDIFF} / \mathrm{v} \text {, }
$$

where HDIFF is the total distance traveled by the cursor between keystrokes. Once the TIME is calculated, the vertical representation is derived from the scaling factor. This vertical scaled number is used to calculate the relative speed per unit line from Equation 1. Finally, using Equation 1 , the total relative distance for each line is calculated and graphed.

\section{Picture Buffer}

The cursor movements in the picture buffer are controlled by the REACT module. Since each line of the HIRES screen is represented by 40 bytes of 7 bits each, the cursor position divided by seven is the byte offset and the position modulus 7 is the bit offset from the start of the line.

To center the cursor, the line position start address +2 bytes is used as the base. This is why references to the $\mathrm{X}$-position in the PASCAL code are 14 dots larger than the $\mathrm{X}$-position referenced in assembly code.

\section{APPLICATIONS OF THE PROGRAM}

The program described in this paper has been successfully used to demonstrate and investigate several phenomena within the area of motor learning and control. These are: (1) the acquisition of tracking performance using a compensatory tracking task, (2) the improvement in acquisition rate when increasing demands are placed upon the subject by decreasing the region of permissible error, (3) the differences in learning and performance that are evident when tracking time (trial time) is increased, (4) the development of response and perceptual anticipatory behavior after practice at a perceptual motor timing task, and (5) the incidental learning of various temporal rhythms, using the response of alternate finger tapping.

\section{REFERENCES}

Apple II Reference Manual for the Ile. (1982). California: Apple Computer, Inc.

PEw, R. W. (1966). Acquisition of hierarchical control over the temporal organization of a skill. Journal of Experimental Psychology, 71, 764-771.

(Manuscript received March 14, 1986; revision accepted for publication May 27, 1987.) 